\title{
CONFLICTOS, DIÁlOGO Y ACCIÓN COLECTIVA CON ENFOQUE TERRITORIAL: NUEVAS FORMAS DE PENSAR LA RELACIÓN COMUNIDAD-EMPRESA-ESTADO
}

\author{
Patricio Carvallo* \\ Guillen Calvo**
}

\section{Resumen}

El desarrollo de proyectos industriales de alto impacto basados en la explotación de recursos naturales en territorios rurales de América Latina implica numerosos desafíos, entre los cuales se encuentra el aumento de los escenarios de conflictividad y las dificultades enfrentadas por el conjunto de actores para implementar mecanismos que permitan transformar posibles conflictos en oportunidades de acción colectiva. En muchos casos, la conducta de los actores en el territorio es guiada por una lógica reactiva y enfoques transaccionales a corto plazo que no permiten que los escenarios de diálogo generen los resultados esperados. Este artículo busca abrir una discusión sobre las tipologías y las causas de la conflictividad existentes, y sobre nuevas formas de pensar los procesos de diálogo desde el entendimiento de la intencionalidad de los actores, estableciendo un posible itinerario entre la transformación de conflictos, el diálogo y una acción colectiva orientada al desarrollo territorial sostenible.

Palabras clave: diálogo multiactor; acción colectiva; conflictos; enfoque territorial; confianza; capital social.

* Máster Científico en Desarrollo rural por la Universidad de Córdoba, España. Director País para Insuco, Quito (Ecuador). [patricio.carvallo@insuco.com]; [https://orcid.org/0000-0002-0161-4106].

** Máster en Geografía y prácticas del desarrollo. Director General para América Latina y el Caribe de Insuco, Bogotá (Colombia) y presidente de la fundación Diversidad y Desarrollo, Montpellier (Francia). [guillen.calvo@ insuco.com]; [https://orcid.org/0000-0003-3032-8544].

Recibido: 24 de junio de 2019 / Modificado: 9 de septiembre de 2019 / Aceptado: 16 de septiembre de 2019

Para citar este artículo

Carvallo, P. y Calvo, G. (2020). Conflictos, diálogo y acción colectiva con enfoque territorial: nuevas formas de pensar la relación comunidad-empresa- Estado. OPERA, 26, 17-36.

DoI: https://doi.org/10.18601/16578651.n26.03 
CONFLICTS, DIALOGUE AND COLECTIVE ACTION WITH A TERRITORIAL APPROACH: NEW WAYS OF THINKING THE COMMUNITY - BUSINESS - STATE RELATION

\section{Abstract}

The development of high impact industrial projects based in natural resources extraction in rural territories of Latin America involves a number of challenges. Among them stand the increase of conflict settings and the increase of difficulties for territorial stakeholders to implement mechanisms to transform these conflicts into collective action opportunities. In many cases, the stakeholders are influenced by a short-term reactive logic characteristic of transactional approaches, which does not allow proper results from a dialog scenario.

This article seeks to generate a discussion about typologies and causes of existing conflicts in high impact industries, new forms of thinking the conversation about the intentionality that stakeholders have for it, and establishing a possible itinerary between conflict transformation, dialogue, and a collective action oriented to sustainable development in the territory where the projects are implemented.

Key words: Conflicts; dialog and collective action with territorial approach; new ways of thinking community-business-State relations.

El conflicto es parte de la "condición humana", y está presente en la vida social y en las relacio- nes entre comunidades, pueblos, empresas y Estados. Si bien existen múltiples definiciones de lo que es un conflicto, se trata de un concepto ambiguo y dinámico, y la forma en que se lo caracteriza condiciona en gran medida la manera en que será abordado.

Según autores como Lederach (1996, 2007), el nuevo paradigma en la gestión constructiva de conflictos sociales evoluciona desde la idea de "resolución al marco de la transformación”. Desde una perspectiva constructivista, el conflicto se entiende como una divergencia de intereses, o la creencia de que las aspiraciones de las partes no pueden alcanzarse simultáneamente (Pruitt, Rubin y Kim, 1994). Un conflicto surge, entonces, cuando dos o más actores, en una relación de interdependencia, no están de acuerdo sobre la distribución de recursos materiales o simbólicos, y actúan basándose en estas supuestas incompatibilidades (Lederach, 1996). En otras palabras, el conflicto no se construye solo por "lo que sucede", sino por el significado que los seres humanos atribuimos a "lo que sucede". Estos significados están basados en nuestra cultura, nuestras historias de relacionamiento, en la base de experiencia y conocimiento que tenemos acumulado.

En este contexto, un conflicto socioambiental podría ser entendido como "una disputa que involucra el uso, acceso y propiedad de recursos naturales y servicios ambientales por grupos de actores quienes tienen diferentes poderes e intereses, y defienden diferentes concepciones con respecto a la gestión de los bienes públicos" (De la Cuadra, 2015, p. 29). Desde esta perspectiva, el conflicto posee algunos componentes distintivos, entre otros, la 
relación de interdependencia entre las partes, es decir, ninguna tiene suficientes recursos como para alcanzar sus objetivos de forma unilateral.

Lo anterior implica entender el conflicto desde la construcción de significados sobre acciones o eventos y la acción en función de esa construcción que realizan actores interdependientes. Es decir, para que exista un conflicto social se estará frente a un asunto que generará percepción, actitud ante esa percepción y comportamiento entre personas o grupos que están relacionados entre sí (Guzmán, 2016).

Considerando esto, la naturaleza de los conflictos socioambientales puede tener múltiples significados en función de los actores que los protagonicen y es multidimensional en la medida de que sean específicos a las dinámicas territoriales en donde radica y se identifica cada comunidad. Este reconocimiento es un primer paso hacia una gestión de estos conflictos para transformarlos en oportunidad para el desarrollo democrático, lo que supone poner el foco no solo en la lucha visible sino en las causas relacionales, institucionales, estructurales y culturales que llevan a los actores sociales a confrontarse alrededor de derechos e intereses contrapuestos (Tapia, Lumerman, Portilla y Spadoni, 2009).

Ahora bien, estos conflictos socioambientales son recurrentes en proyectos industriales de alto impacto, entendidos como aquellos proyectos cuya actividad tiene el potencial de alterar, no solo factores socioeconómicos y ambientales aislados, sino también las dinámicas territoriales (gobernanza, desarrollo productivo, valores sociales y cultura, migración y salud, entre otros) donde estos se implementan. Así, en este tipo de proyectos (minería, generación de energía, petróleo y gas, infraestructura y agroindustria, entre otros), los determinantes de los conflictos no radican únicamente en aspectos ambientales y económicos, sino también en aspectos sociales y de gobernanza. Por tanto, el análisis de los mismos debe incluir múltiples factores como la historia de la comunidad en relación con la presencia de proyectos extractivos, la etapa del ciclo del proyecto en el que se encuentre, el tipo de empresa que promueve el proyecto, sus modos de relacionamiento, y las historias individuales y colectivas de los actores que lideran la gobernanza del territorio, entre otros factores.

Lo anterior sugiere que hablar de conflictos socioambientales de forma genérica es poco operativo y no refleja la diversidad de situaciones observadas a nivel local en el contexto de proyectos industriales de alto impacto. Según De la Cuadra (2015, p. 30), dentro de las causas de los conflictos socioambientales se pueden encontrar los propósitos que diferentes actores y agentes económicos (individuos o empresas) asignan al uso de la tierra y los recursos naturales, que a su vez se sustentan en valores culturales de las sociedades y que, a su vez, se basan en una construcción particular de modernidad que valora el conocimiento científico occidental.

Es por esto que, a partir del trabajo realizado por los autores en más de diez proyectos industriales en Colombia, Perú, Ecuador, Chile y Honduras, se han logrado identificar patrones comunes en el análisis de los conflictos que permiten identificar una distribución por tipologías y causas. Analizar causas permite 
entender los determinantes de posibles conflictos y, por ende, incidir en la transformación del mismo desde sus significados y no solo desde su materialización. Cabe destacar que esta propuesta de tipologías no busca ser una categorización definitiva, sino una aproximación para alimentar el análisis y la investigación en torno a los determinantes de conflictos socioambientales en el contexto de proyectos industriales de alto impacto.

Esas causas pueden distribuirse en tres niveles que generalmente se interrelacionan y se alimentan entre ellas en las raíces de los conflictos: 1) temáticas, 2) coyunturales y 3) estructurales (figura 1).

A continuación, se citan algunas causas de carácter temático con mayor presencia en los conflictos entre comunidad, empresa y Estado.

- Conflictos en donde prevalece la problemática ambiental: se presenta una afectación directa sobre los recursos naturales, tales como contaminación del agua, del aire o la tierra. Algunos ejemplos son derrames, deforestación, delimitación de áreas de interés biológico (p. ej.: páramos, zonas de parques naturales), afectaciones por levantamiento de polvo, contaminación de aguas por residuos industriales, etc.

- Conflictos en donde prevalece la problemática sociocultural: se presenta una afectación directa sobre las conductas socioculturales del territorio producto de la llegada de nuevos habitantes y trabajadores, o el aumento de los ingresos asociados a las oportunidades laborales que pueden derivar en problemas de inse- guridad y en el aumento de conductas de riesgo como drogadicción y alcoholismo, además de las posibles afectaciones a la identidad cultural.

- Conflictos en donde prevalece la afectación al "contenido local": el conflicto se da en el contexto de problemáticas asociadas a la falta de oportunidades de empleo o de contratación de bienes y servicios locales. Generalmente, esas problemáticas tienen sus orígenes en la percepción de acceso a los beneficios derivados de un proyecto por parte de actores territoriales. Esto involucra principalmente a comunidades, pero también a gobiernos locales y nacionales que buscan acceder a los recursos derivados de los proyectos.

- Conflictos asociados a una competencia entre actores sobre el uso de la tierra: que van desde la delimitación de concesiones, a procesos de expropiación, reasentamiento, presencia de actores extractivos informales en las concesiones de las empresas, etc.

Diferentes situaciones observadas por los autores de este artículo, en el contexto de proyectos industriales en países de Latinoamérica, muestran que en muchos casos los conflictos se presentan, en primera instancia, como consecuencia de una falta de acceso a ciertos beneficios derivados de los proyectos, tales como empleabilidad, contratación de bienes y servicios (los llamados temas de "contenido local"), más que de aspectos asociados a temas ambientales o socioculturales. Si bien la relación entre problemáticas ambientales o lógicas de defensa del territorio frente al desarrollo de 
proyectos industriales ha sido analizada por el mundo de la investigación y grupos de la sociedad civil, esta relación entre expectativas, beneficios inducidos por proyectos industriales y conflictividad carece aún de trabajos de revisión y análisis que permitan entender las diferentes trayectorias, representaciones y formas de transformación que han tenido estos conflictos.

Por otra parte, entre las múltiples causas coyunturales que pueden servir de detonador de los conflictos se encuentran:

- Falta de información sobre los proyectos: a menudo, la oposición a los proyectos de alto impacto no está ligada a la protección del medio ambiente o de la tierra, sino a la necesidad de las personas de controlar sus recursos y decidir sobre el curso de sus vidas (Damonte, Glave y Kuramoto, 2014). Cuando las comunidades locales no son informadas e involucradas oportunamente, antes y durante la llegada de las empresas al territorio, pero también durante los cambios de fase de los proyectos (exploración, construcción, explotación, cierre) esto puede, per se, generar resistencia hacia dichas compañías y sus proyectos. Ciertas empresas consideran que los permisos gubernamentales son elementos suficientes para iniciar sus trabajos y descuidan la necesidad de establecer un relacionamiento oportuno y adecuado con las comunidades y sus diferentes grupos de interés, más allá de los procesos de licenciamiento establecidos en el marco legal. La falta de información suele traducirse en percepciones y rumores que pueden desencadenar fuertes tensiones.

- Expectativas de beneficios no satisfechas: la llegada de grandes empresas genera en las comunidades locales un nivel alto de expectativas en materia laboral, de servicios sociales y de infraestructura (relación con la causa temática de contenido local). A menudo, y frente a la relativa o total ausencia del Estado en esas zonas, se considera desde la comunidad que las empresas deben asumir este tipo de inversiones. La falta de una comunicación fluida entre la empresa y la comunidad, o la búsqueda de aceptación social por parte de la compañía en sus fases iniciales, puede llevar a que estas expectativas no sean adecuadamente confrontadas con las perspectivas reales. En consecuencia, cuando los proyectos no responden a la velocidad esperada por la comunidad, pero sí en función de las necesidades del proyecto, y la comunidad no percibe los supuestos impactos positivos presentados inicialmente por las empresas, se generan frustraciones y posibles tensiones.

- Coyuntura política: el apoyo o la oposición a los proyectos industriales de alto impacto suele ser un argumento de campańa y marcador político que agudiza directa o indirectamente ciertos conflictos específicos que se dan a partir de la diferencia de incentivos entre las partes.

- Coyuntura relacionada con el modelo o tipo de relacionamiento que la empresa desarrolla con sus grupos de interés: en la medida que a lo largo del tiempo las empresas cambien las estrate- 
gias pero también los profesionales que gestionan las áreas de relacionamiento, lo que puede variar a corto y mediano plazo las líneas de gestión social o llevar a profesionales a asumir posiciones que deriven en tensiones o conflictos con las comunidades.

Finalmente, entre las causas estructurales que promueven escenarios de conflictividad, se encuentran:

- Vulnerabilidad de las zonas donde se desarrollan los proyectos: los factores antes descritos se combinan a menudo con la situación de vulnerabilidad que suele caracterizar las zonas rurales donde se ubican muchos proyectos de alto impacto. Eso se traduce en múltiples carencias en el acceso a servicios básicos, infraestructura, la permanencia de problemáticas sociales fuertes y bajos niveles educativos que afectan el tejido social y económico de los territorios. Así, la percepción de que una gran riqueza se produce en escenarios de gran pobreza, sin transformar la calidad de vida de los habitantes es mayor (González, 2016), por lo que las expectativas que puede generar la llegada de un proyecto industrial en este tipo de contextos se ven agudizadas por la situación social previa a la llegada del este, y refuerzan la presión que ejercen el conjunto de actores territoriales, desde la comunidad hasta los entes territoriales, para que el proyecto pueda cerrar las brechas sociales que se han venido acumulando a lo largo del tiempo.
Aunque la llegada de los proyectos industriales genera rentas que pueden potenciar el desarrollo territorial, es imposible pensar que un proyecto, por más grande que sea, pueda resolver por sí solo situaciones de vulnerabilidad social, económica e institucional acumuladas durante décadas. En general, estos recursos no suelen ser suficientes o se generan tensiones y desacuerdos en torno a la forma de su distribución y utilización, por lo que muchos conflictos están asociados al deseo de las comunidades locales y los actores institucionales de obtener una mayor porción de la renta generada por dichos proyectos.

- Falta de institucionalidad: muchas zonas donde se desarrollan esos proyectos se caracterizan por una ausencia histórica del Estado y de sus instituciones, con niveles muy bajos de inversión en servicios sociales básicos u obras de infraestructura. Eso lleva a menudo a la población a esperar que la empresa supla este déficit y que, de alguna forma, se sustituya el rol del Estado. La empresa, a su vez, espera que sea el Estado quien genere nuevos proyectos de desarrollo, en particular, en lo que se refiere a proyectos de infraestructura y de acceso a servicios básicos a partir de las nuevas rentas generadas. A la falta de capacidades y la ineficiencia se puede sumar la corrupción, lo que agrava la situación. Esto implica que cuando emergen conflictos sociales en el territorio o, en general, cuando se busca promover espacios de diálogo entre múltiples actores, la falta de credibilidad, neutra- 
lidad y capacidad de las instituciones públicas para actuar como mediadores, o su intervención inequitativa en favor de uno de los actores, suele ser un detonante adicional del escalamiento del conflicto. Según Watkins (2017), los conflictos que escalan confrontaciones violentas son propios de países que carecen de una capacidad institucional para la gestión efectiva de los mismos. Además, existe una correlación entre países con baja capacidad institucional y gobernanza y la magnitud de las expresiones de conflicto.

En la práctica, esas tipologías no son categorizaciones estrictas, sino que la mayoría de los conflictos tienen sus orígenes en una combinación de esas tres categorías de causas. Entender las dinámicas del territorio de acuerdo con el conjunto de esos factores y tipologías puede servir para analizar los riesgos de conflictividad latentes en un territorio determinado, prevenir los escenarios de conflictividad buscando escenarios de diálogo que permitan abordar el conjunto de causas en espacios donde participen los actores relevantes de acuerdo con cada causa identificada, y poner los conflictos socioambientales en perspectiva abordándolos desde sus causas y tipologías, y no solo desde sus manifestaciones.

\section{DIÁLOGO Y CONCERTACIÓN EN EL CONTEXTO DE PROYECTOS DE ALTO IMPACTO}

A diferencia de la resolución o la gerencia de conflictividad, la idea de transformación

FIGURA 1. TIPOLOGÍAS POR CAUSAS DE CONFLICTOS ENTRE COMUNIDAD-EMPRESA-ESTADO

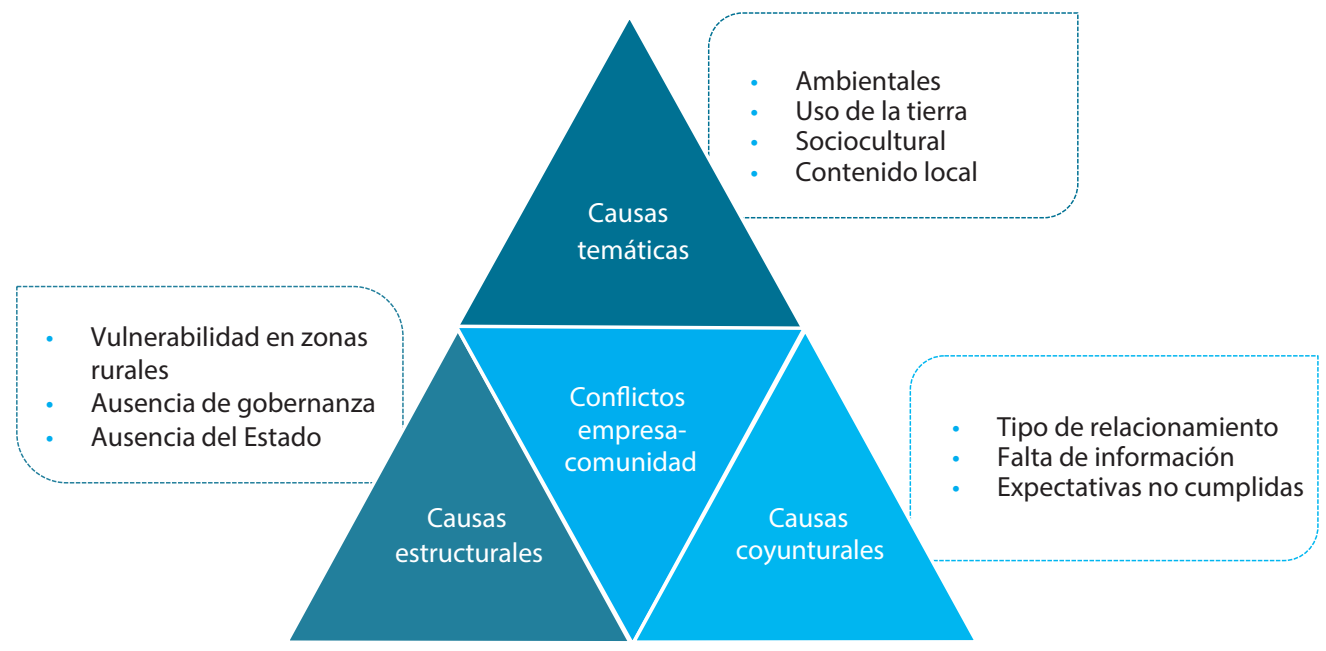

Fuente: elaboración propia. 
en su abordaje no sugiere que simplemente eliminemos o controlemos el conflicto, sino que apunta de manera descriptiva a un entendimiento de su naturaleza inherentemente dialéctica. Es un fenómeno que transforma eventos, las relaciones atravesadas por el conflicto, de hecho, cambia a sus propios protagonistas (Lederach, 1996). En otras palabras, transformar implica que el conflicto genere cambios a diferentes niveles de escala. Que el cambio en cuestión sea constructivo o destructivo, democrático o autoritario, depende en gran medida de la calidad del liderazgo que es aplicado a su abordaje y de los espacios existentes para abordarlos. De allí el interés de poner en perspectiva el análisis de los conflictos con las estrategias que los actores locales van a desarrollar para prevenirlos, transformarlos $y$, eventualmente, avanzar hacia escenarios de cooperación y construcción colectiva. Esto permite cuestionar y analizar las diferentes formas de pensar y entender el diálogo en el contexto de proyectos industriales de alto impacto como mecanismos para la transformación de conflictos socioambientales. El análisis de trabajos realizados sobre las dinámicas de concertación multiactoral, que incluyan contextos diferentes al latinoamericano, nos permite poner en perspectiva nuestro análisis sobre los procesos de diálogo.

En ese sentido, la concertación como unidad de análisis ha dado lugar a diferentes trabajos de investigación por autores entre los cuales cabe destacar a Jean Eudes Beuret, quien ha venido analizando las dinámicas de concertación entre actores en diferentes escenarios incluyendo la conformación de áreas protegidas y mecanismos de participación social asociados a estudios y procesos de licenciamiento ambiental. Como se puede ver en la figura 2, esta, ya sea elegida o impuesta, siempre responde a una combinación dosificada de forma conjunta de tres objetivos: "el objetivo de informar, el de generar aceptación y el de coconstruir", en donde cada uno de esos objetivos responde a "intenciones" y "tipos" de diálogo diferentes (Beuret, 2012).

De esta manera, se puede decir que generar un entendimiento común desde un inicio por parte de los promotores de los espacios de concertación o de diálogo sobre sus objetivos es fundamental. No es la diversidad de objetivos la que plantea problemas, sino la diferencia de representaciones y expectativas que pueden existir de parte de los actores representados. En ciertos casos, el desgaste o fracaso de los espacios de diálogo viene asociado a esas diferencias de representaciones y expectativas entre actores frente a los objetivos de los espacios de diálogo o sobre los mecanismos desarrollados para cumplir con esos objetivos.

Bajo ese entendimiento, es importante entender las diferencias entre las representaciones existentes detrás de distintos mecanismos de participación, tales como consulta, negociación, concertación y diálogo, donde algunos autores ubican los conceptos de consulta, concertación y negociación en un mismo continuum (Beuret, 2012).

En lo que se refiere específicamente al diálogo, los recientes trabajos de análisis desarrollados por grupos multiactorales, como el Grupo de Diálogo sobre Minería (GDIAM) en Colombia, aportan elementos interesantes para esta reflexión. Ese grupo identifica dos tipos de diálogo que, al ser definidos, comparten 
FIGURA 2. LOS OBJETIVOS DE LA CONCERTACIÓN: TRES POLOS Y TRES GRADIENTES

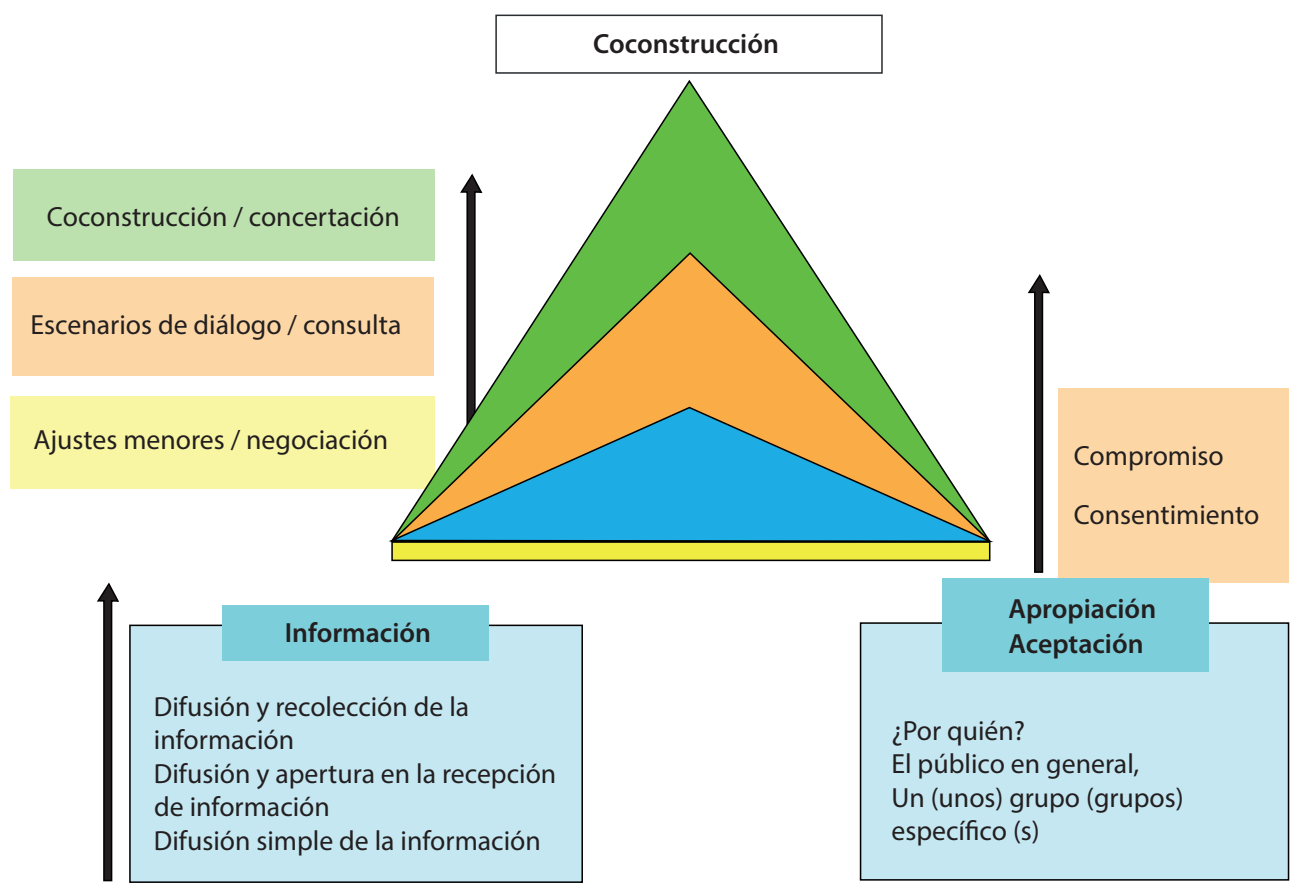

Fuente: Beuret (2012).

características presentadas en la definición de concertación de Beuret. Por una parte, identifican el "diálogo para la negociación”, que se caracteriza porque permite,

... de un lado, procesar y tramitar el conflicto para evitar que llegue a comportamientos violentos, y, de otro, encontrar soluciones -al menos de corto plazo- que reduzcan las tensiones y el tono del antagonismo $[\ldots]$ en los que el consenso implica ganancias y pérdidas para ambas partes, desde el supuesto de que lo conseguido como logro por un actor puede significar una pérdida para los otros actores. (GDIAM, 2018, p. 9)
Por otra parte, se identifica el "diálogo estratégico" que

... busca construir consensos estratégicos de mediano y largo plazo, que pueden en adelante orientar la conducta de las partes involucradas, en la medida en que cada una de ellas considera que el acuerdo logrado la beneficia. [...] El diálogo estratégico no busca negociar intereses, sino generar confianzas mutuas para la construcción de acuerdos de utilidad para las partes e, incluso, para otros actores de la sociedad. Su producto final es la formulación de visiones compartidas sobre asuntos de trascendencia para las partes. (GDIAM, 2018, p. 9) 
En ese sentido, en el "diálogo estratégico" prevalece una intencionalidad descrita por Dupont (2006) como "concertativa" y basada en principios de "cooperación", lo que nos lleva a calificar a esos procesos como diálogos orientados hacia formas de acción colectiva. Beuret (2006) y Beuret y Cadoret (2011) diferencian el término de concertación del de consulta, e indican que el interés de ese tipo de mecanismos radica sobre todo en el hecho de construir juntos objetivos comunes.

De esta manera, se puede entender que la concertación va más allá de una simple consulta, ya que trata de adaptar las decisiones a las necesidades de los actores ajustando los intereses de cada uno de ellos (Beuret, 2012). Bourque (2008), otro autor que ha analizado el concepto de concertación insiste en el hecho de que esta es una forma de cooperación. Es decir, un proceso colectivo de coordinación basado en una relación estructurada y duradera entre actores sociales autónomos que aceptan compartir información, discutir problemas específicos o temas (por problema o por territorio) para acordar objetivos y acciones comunes que puedan o no derivar en alianzas.

Es más, Dupont (2006) insiste en la necesidad de entender las "intenciones" detrás de los ejercicios de negociación y concertación para poder analizarlos como un proceso no linear y de acuerdo con lo que podríamos calificar como itinerarios de concertación o, en este caso, itinerarios de diálogo. Este mismo autor afirma que hay negociaciones que, por esencia, son conflictivas, cuando otras por esencia serán cooperativas o distributivas y que, por ende, resultan de una intención diferente. En una negociación conflictiva o distributiva, cada uno trata de construir un equilibrio de poder que sea favorable para él y lo aprovecha para imponer sus preferencias (diálogo para la negociación de acuerdo al GDIAM). En la negociación "concertativa” definida por Dupont (2006), los actores están de acuerdo en cooperar con el otro para construir juntos una solución mutuamente aceptable. Este tipo de negociación está cerca de la concertación definida por Beuret (2012) y del diálogo orientado a la acción colectiva, caracterizada por el hecho de construir visiones, objetivos, decisiones y proyectos en conjunto.

Para Dupont (2006), la clave está, por tanto, en entender la intención detrás de cada ejercicio de diálogo. Si la intención es cooperativa, incluso durante episodios de conflictos, evitaremos el uso de estrategias demasiado agresivas que transformarían el diálogo hacia una negociación conflictiva y buscaremos volver a la intención fundamental de la cooperación o la acción colectiva.

En este contexto, es importante preguntar ¿cuál es la intencionalidad detrás del diálogo? El diálogo entre actores para la transformación de conflictos socioambientales de proyectos industriales de alto impacto no asegura un proceso "plano", sin picos de intensidad (altos o bajos), en donde se agudizan las diferencias entre los incentivos de cada actor que es concertado. El diálogo pierde efectividad si no es asociado a una "intencionalidad" u objetivos claros y, por tanto, se expone a las voces que consideran que este es hoy en día un concepto desgastado, que no genera resultados, sino que se limita a reproducir los intereses de los actores con mayor poder e influencia en el territorio. Existen numerosos ejemplos de espacios de concertación y diálogo en el contexto de pro- 
yectos industriales de alto impacto en distintos países de Latinoamérica (Chile, Perú, Argentina, Colombia y Ecuador, entre otros) que han fracasado y, como consecuencia, han llevado al debilitamiento y desgaste de esos espacios y, por ende, del mismo concepto. Estos casos se han enfocado principalmente en atender de forma reactiva escenarios de conflictividad desde una perspectiva, según Walton y MacKersie (1965), de diálogo para la negociación o negociación distributiva, en donde la intencionalidad justamente está en evitar una escalada de tensiones y expresiones de "violencia" para lo que se buscan resultados a corto plazo.

Como se observa en la figura 3, cada etapa significa una intencionalidad diferente. En el primer caso, el diálogo es meramente informativo, a fin de pasar posteriormente a un diálogo para la sociabilización donde no solo se informa, sino también se busca tener alguna forma de retroalimentación. En el caso del diálogo para la acción colectiva o "diálogo estratégico" se busca involucrar a los actores del territorio para desarrollar una visión compartida de las oportunidades que presenta el proyecto para el territorio. Finalmente, sin que sea linear como cualquier proceso social, la ruta del diálogo llega a su madurez cuando el diálogo para la acción colectiva logra alinear objetivos de cooperación y coconstrucción a más largo plazo, buscando incentivos para que los actores territoriales aborden las causas más coyunturales y estructurales de la conflictividad territorial, a fin de generar herramientas de planificación y gestión desde una visión conjunta del espacio que todos comparten (su territorio).

Introducir la noción de proceso en el análisis de los ejercicios de diálogo permite plantearlo como un fenómeno no linear. El diálogo nunca es un camino corto para llegar a un acuerdo, implica conflictos y tiempos de construcción o regresión durante una ruta en la cual no se pueden conocer los contornos antes de que se construyan, debido a las interacciones entre los participantes. De esta forma, la noción de cooperación, asociada a ciertas formas de diálogo o de concertación, no significa que no haya tiempo para la tensión o incluso el conflicto (Beuret, 2012).

Tanto Beuret (2012) como Dupont (2006) reconocen que la concertación o el diálogo se sustentan en tres grandes principios comunes

FIGURA 3. RUTA DEL DIÁLOGO

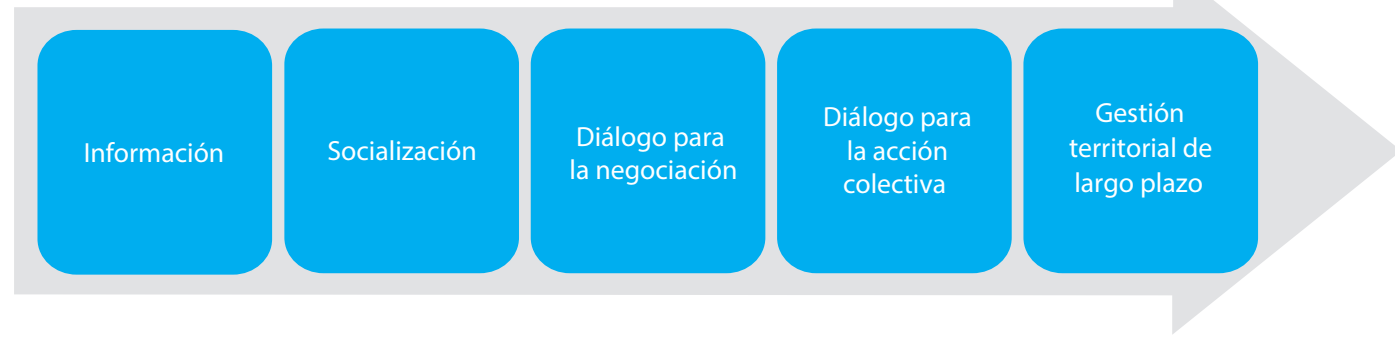

Fuente: elaboración propia. 
a sus diferentes manifestaciones: 1) cooperación, 2) construcción o acción colectiva, y 3) proceso. El uso del término "proceso" apunta a distinguirse del término "procedimiento", que tendería a reducir la consulta a instancias, pasos y formalidades que se deben respetar, mientras que esos procesos siempre integran contingencias, tiempos que pueden ser largos y una cierta informalidad. Esa dimensión de proceso, asociada a la dimensión de diálogo y de transformación de conflictos, refuerza la necesidad de que la intencionalidad que los guíe pueda mantenerse en el tiempo, más allá de las coyunturas políticas y sociales que conocen todos los territorios.

\section{(AUTO)GOBERNANZA EN EL CONTEXTO DE CONFLICTOS SOCIOAMBIENTALES}

Gobernanza y participación de un tercer actor son elementos clave que permiten garantizar continuidad en la intencionalidad de un diálogo orientado a la acción colectiva y las transformaciones territoriales. Si vinculamos el análisis de los conflictos desarrollado en la primera parte de este artículo, con el análisis de las diferentes formas de diálogo, es evidente cómo esos conceptos se interrelacionan de forma directa con el de gobernanza y, en particular, con el de gobernanza territorial.

Como lo plantea Jorquera (2011), la gobernanza territorial y local implican una nueva manera de relacionar la acción pública y las formas en que esta es aplicada en los territorios, gracias a la emergencia y puesta en práctica de innovadoras formas compartidas de planificación y gestión de las dinámicas territoriales, a fin de acordar visiones comunes de todos los actores y niveles concernientes en pos del futuro del territorio. Por tanto, la participación de la población en los asuntos públicos y, concretamente, en las decisiones que impactan a los territorios, constituyen los requisitos relevantes para la nueva gobernanza.

La asimetría de información, relacionada con la falta de transparencia y de voluntad a la hora de comunicar por parte de actores territoriales sobre temas potencialmente gatillantes de conflictividad relacionados con proyectos de alto impacto, genera y agudiza conflictos de esta índole. La ausencia de una gobernanza con una intencionalidad orientada hacia la "inclusión" de los actores territoriales, con especial foco en aquellos que se encuentran en condición de vulnerabilidad (mujeres, jóvenes, adultos mayores, etc.), dificulta y hasta obstaculiza el conocimiento, el diálogo y el cumplimiento de las expectativas que los actores clave tienen en torno al proyecto.

Para que los procesos de gobernanza sean incluyentes es necesaria la participación temprana de los actores territoriales para impulsar espacios de diálogo en los que existan interacciones sociales basadas en confianza y normas de reciprocidad, redes y formas de participación civil, y reglas formales e informales o instituciones. Estos sistemas, incluso de autogobierno, tienen mayor posibilidad de mantenerse en el tiempo, siempre y cuando "la estructura del proceso que transforma las acciones individuales en resultados (y la magnitud de las retribuciones obtenidas por los individuos) [tenga] un efecto obvio en la probabilidad de que se dé una acción colectiva” (Ostrom, 2003, p. 197). A pesar de que 
muchos factores contribuyen a la mantención en el tiempo de un proceso de gobernanza, la mayoría están relacionados -e incluso enfocados específicamente- con los incentivos e intenciones que, a su vez, están relacionados con las expectativas de los actores territoriales. Lo anterior abre la pregunta sobre la sostenibilidad de los espacios de diálogo que son parte de procesos no lineales, en parte impredecibles, y que requieren continuidad en el tiempo. Para mantenerse en el tiempo esos espacios deben seguir respondiendo a las expectativas de los actores territoriales y, consecuentemente, alinearse con sus intenciones generando incentivos para su participación efectiva.

La creación de nuevos incentivos que puedan ser monitoreados por los actores territoriales organizados que son parte del proceso de gobernanza va generando cambios positivos hacia una autogobernanza e impulsa y fortalece una acción colectiva, siempre y cuando estas acciones cumplan las expectativas de los actores territoriales y generen confianza en el proceso. Estos cambios, además, buscan el empoderamiento de los actores clave del proceso, para que sean ellos mismos quienes hagan cumplir las reglas formales e informales que se han establecido con anterioridad. De esta manera, la red de relaciones dentro del espacio de (auto) gobernanza, y la vinculación de algunos de los actores territoriales con otras personas que están fuera del espacio de gobernanza condicionará el éxito de la acción colectiva para la transformación del conflicto en una oportunidad para el desarrollo territorial.

Ejemplo de esto es que en territorios donde las comunidades han debido enfrentar una serie de factores que han aumentado su sensibilidad y el rechazo por los proyectos industriales, como son las malas prácticas ambientales, han organizado y tomado poder de negociación, fortaleciendo su (auto)gobernanza a la hora de enfrentar nuevos proyectos de alto impacto. Es decir, cuanto más historia tenga una comunidad-territorio sobre relacionamiento comunidad-empresa-Estado, mayor exigencia socioambiental va a existir para los proyectos industriales, yendo más allá de las preocupaciones particulares en torno a temas como la inserción de mano de obra y contratación de bienes y servicios para responder a intereses comunes que van evolucionando en el tiempo.

Muchos de los conflictos comunidad-empresa-Estado se relacionan o se ven agudizados por el tipo de relación preexistente entre la empresa y los actores en el territorio. Esa relación no es estática y puede ir variando en función de ciertas variables de gestión como la definición de estrategias o políticas de responsabilidad social empresarial (RSE) dentro de las empresas, el cambio o la ausencia de profesionales encargados de impulsar estas estrategias o de cargos directivos, accionistas en las empresas, los cambios de liderazgos sociales o políticos a nivel local que inciden en el tipo de interlocución que mantiene la empresa con los actores territoriales, e incluso cambios por situaciones externas al territorio como las condiciones del negocio. Todas estas acciones buscan generar mayor confianza entre los actores que participan de la gobernanza del territorio donde se implementa el proyecto de inversión a gran escala, pero, en muchas ocasiones, estos esfuerzos se dan a partir de diálogos bidireccionales, perdiendo la posibilidad de construir capital 
social que, según Putnam et al. (1993, p. 167) son "aspectos de la organización social, tales como confianza, normas y redes, que pueden mejorar la eficiencia de una sociedad al facilitar la acción coordinada" (citado por Ostrom, 2003, p. 166).

En este punto es donde entendemos la importancia de la participación de un actor facilitador y orientador que acelera la evolución del tipo de relación comunidad-empresaEstado a través de esa puesta en marcha de un diálogo para la acción colectiva que, además de la generación de los espacios de diálogo, se encarga de ir posicionando la relevancia de cumplir las reglas y los compromisos adquiridos.

De la misma manera, el actor facilitador del diálogo tiende a ser el garante y custodio de esa intencionalidad que permite orientar el proceso de diálogo hacia objetivos de corresponsabilidad y de acción colectiva. Además, es el encargado de gestionar la materialización de esa visión conjunta (inicialmente compromisos, para pasar a proyectos y continuar con un proceso de planificación compartida). Como lo plantean Dziedzicki y Michel (2017) en su análisis sobre el rol del "tercer actor garante" de los procesos de concertación, su objetivo es garantizar, a través de la intervención de un tercero considerado neutral e independiente, que el mecanismo participativo implementado se base en información de calidad accesible para la mayor cantidad de personas posible, así como una expresión genuina de las poblaciones involucradas.

El rol de ese tercer actor facilitador y catalizador de procesos, garante del cumplimiento de los objetivos del diálogo alejado de intereses particulares, neutral y legítimo frente a los actores locales, es una figura relativamente nueva en los escenarios de diálogo multiactor a nivel latinoamericano. Ese tercer actor, cuyas responsabilidades y modalidades de intervención pueden diferir de acuerdo con los proyectos y territorios, amerita ser estudiado a través de la movilización de disciplinas como la sociología de la innovación. Casos como el trabajo de facilitación que vienen desarrollando organizaciones como Insuco en el contexto del proyecto extractivo Fruta del Norte en Ecuador (Arratibel, Calvo, Estrada, Guio y Romero, 2019), el trabajo impulsado por Jaime Arteaga \& Asociados en el marco del plan Buriticá vinculado al proyecto aurífero de Continental Gold en Colombia, y el ejercicio de territorialización del diálogo impulsado por el GDIAM en Colombia, son casos novedosos cuyos resultados y lecciones aprendidas deben de ser analizados.

\section{DEL RELACIONAMIENTO \\ TRANSACCIONAL HACIA UNA MIRADA TRANSFORMACIONAL}

Los elementos anteriores apuntan hacia las bases de un diálogo que construya capital social y ciudadanía al impulsar cambios culturales, orientando la atención de los actores involucrados en la (auto)gobernanza del territorio puesta en un aspecto/actor específico, el proyecto industrial de alto impacto, hacia el desarrollo de un espacio compartido, el territorio. De esta manera, procesos de diálogo consolidados bajo un enfoque territorial enfatizan el desarrollo del territorio a través de 
la articulación de actores y la generación de oportunidades compartidas.

En este contexto, y como se aprecia en la figura 4 , se encuentran tres estrategias para la "colaboración continuada" entre comunidadempresa-Estado: 1) la Estrategia transaccional, en donde el relacionamiento empresa-comunidad está basado en la difusión unidireccional de información, en donaciones caritativas y actividades voluntarias de los empleados; 2) la Estrategia transicional, en donde la implementación de actividades de las empresas se da a través de espacios incipientes de diálogo con las partes interesadas de la comunidad, consulta pública, establecimiento de acuerdos básicos de cooperación; y 3) la Estrategia transformacional, en donde la orientación de la participación empresarial se da a través de comportamientos como la corresponsabilidad, la propiedad y la gestión conjunta de proyectos, y la toma de decisiones compartida con los grupos de interés (Bowen, Newenham-Kahindi y Herremans, 2010). Esta evolución retoma, desde una nueva perspectiva alineada con las relaciones entre comunidad-empresa (proyectos industriales de alto impacto)-Estado, los polos y gradientes de la concertación formulados por Beuret y las intencionalidades del diálogo observadas anteriormente.

En Latinoamérica, se podría decir que la estrategia de colaboración continuada más presente en proyectos industriales de alto impacto sigue siendo la transaccional, en donde las empresas basan principalmente su relación

FIGURA 4. AUMENTO DEL NIVEL DE PARTICIPACIÓN DE LAS COMUNIDADES

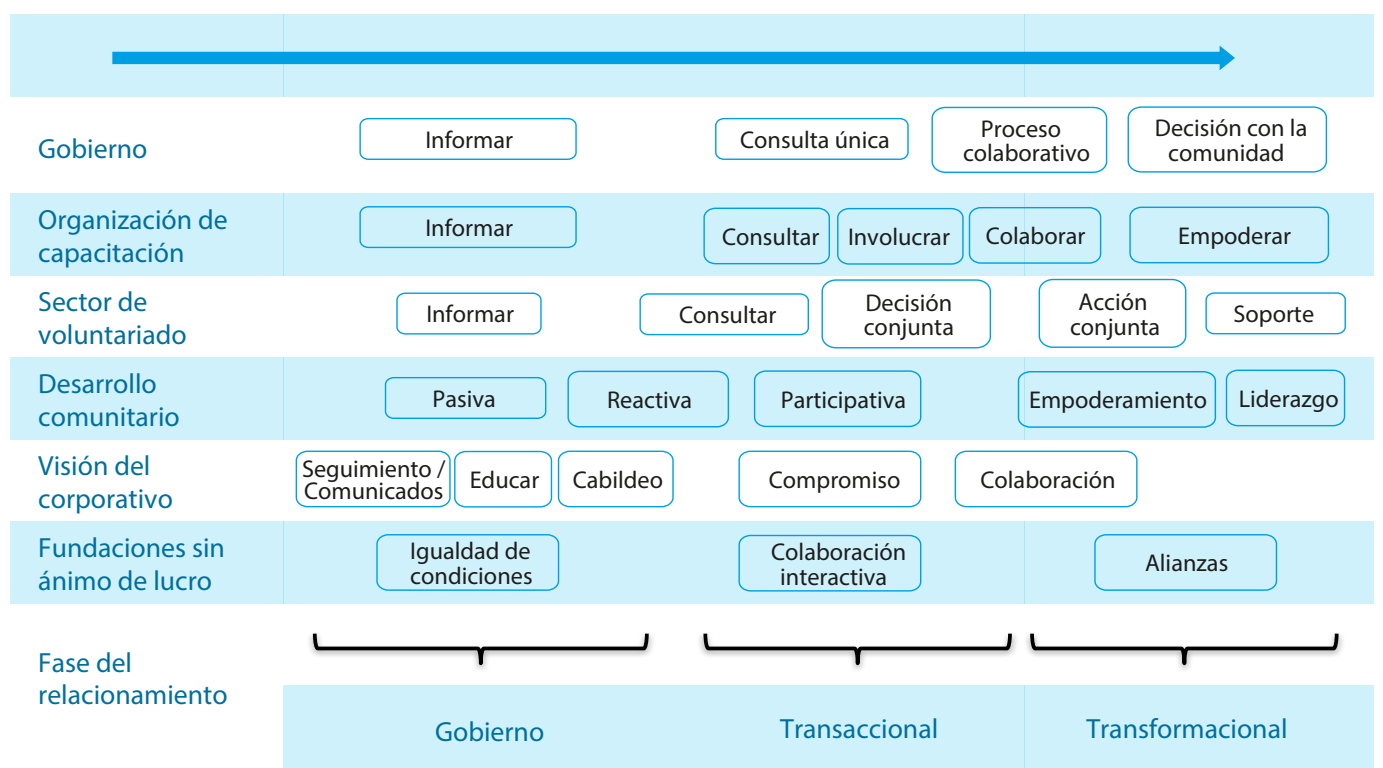

Fuente: basado en Bowen et al. (2010, p. 304). Adaptado por Insuco (2017). 
con los grupos de interés en aportes puntuales de recursos financieros de la empresa (filantropía) sin fundamentos reales de cooperación o de aporte a procesos de gobernanza para el desarrollo territorial. Estos aportes se basan en la atención de necesidades puntuales y no de objetivos compartidos de largo plazo, es decir, se utiliza la inversión social privada como estrategia de viabilización de proyectos y resolución a corto plazo de conflictos. Por tanto, el compromiso transaccional se basa en prevenir, resolver conflictos o desarrollar espacios de diálogo de forma reactiva, sin intención de generar acciones transformadoras que puedan incidir en las múltiples causas de los riesgos observados anteriormente. Este enfoque representa la praxis con la que muchas empresas responden a las expectativas de las comunidades, que legítimamente quieren acceder a los beneficios de los proyectos, pero, además, a las expectativas de las autoridades de gobiernos locales y centrales de acceder a los beneficios que el proyecto les pueda entregar.

De la misma manera que las estrategias de relacionamiento empresa-territorio, los procesos de diálogo deberían transitar de una estrategia transaccional a una transformacional. Esto quiere decir que el diálogo comunidadempresa-Estado debe pasar: 1) de una mirada de "dar de regreso a la comunidad", a la generación de cambio en la sociedad a través de un proceso de integración con la comunidad; 2) de una lógica de donaciones o retribuciones a un enfoque donde la toma de decisiones del proyecto se realiza conjuntamente con la comunidad a partir de mecanismos de cooperación; 3) de una información unidireccional empresa-comunidad a una comunicación recí- proca empresa-comunidad-empresa en donde además participa el Estado; 4) de una interacción ocasional a una acción común y compartida periódica y constante; 5) de procesos de aprendizaje en donde la empresa transfiere el conocimiento a las comunidades, a un proceso de aprendizaje creado e innovado continuamente en conjunto y con el Estado; 6) de un control de proceso llevado exclusivamente por la empresa, a uno en donde los controles son horizontales, colectivos y distribuidos entre una diversidad de actores (Bowen et al., 2010).

\section{CONCLUSIONES: DIÁLOGO MULTIACTOR PARA LA ACCIÓN COLECTIVA, UN ENFOQUE TERRITORIAL PARA LA (AUTO) GOBERNANZA Y SU DESARROLLO}

Según Schejtman y Berdegué (2003), el desarrollo territorial se entiende como un proceso de transformación social, productivo e institucional, en un espacio rural determinado que tiene el propósito de articular competitiva y sustentablemente a la economía del territorio a mercados dinámicos. Además, busca responder a la necesidad de pasar de un desarrollo productivista, sectorial y centralista dirigido a la diversificación económica y territorial, a un modelo posproductivista, que valore todos los componentes del espacio, flexible, integrado y participativo (Cebrián, 2004).

Bajo ese entendido, el territorio no se debe concebir como un espacio físico "objetivamente existente", sino como una construcción social, es decir, como un conjunto de relaciones sociales que rigen y, a la vez, expresan una identidad y un sentido de propósitos compartidos 
por múltiples agentes públicos y privados. De esta forma, se considera al territorio como un producto social e histórico, al estar la sociedad ligada al espacio físico en que se encuentra, y constituirlo mediante un proceso de apropiación en el que evolucionan simultáneamente sistemas económicos, sociales, institucionales, culturales, políticos y ambientales (Sepúlveda, Rodríguez, Echeverri y Portilla, 2003).

Así es como el enfoque territorial de responsabilidad empresarial pretende superar la visión transaccional del relacionamiento entre actores reconociendo la complejidad y diversidad de los territorios rurales y de sus formas de gobernanza para favorecer una visión multidimensional del manejo de los recursos naturales, en que lo medioambiental, lo económico, lo social y lo político-institucional interactúan en el territorio. De esta manera, cuestiona los modelos transaccionales de diálogo en donde la empresa es el centro de atención del mismo, y promueve que esta sea un actor más de la (auto) gobernanza del territorio compartida con otros actores bajo una mirada de cooperación. De esta manera, se podría decir que el enfoque territorial se centra en un "diálogo estratégico" para pasar de una visión transaccional de la participación comunidad-empresa-Estado a una visión transformacional de gobernanza.

Aspectos operativos-estratégicos característicos del enfoque territorial son un conocimiento detallado del entorno; la inclusión y acción colectiva (empresa, gobierno, comunidad y otras entidades); mecanismos de comunicación continuos, oportunos, respetuosos y adaptados; un conocimiento detallado del entorno; una inversión en el fortalecimiento del capital social, humano e institucional, y una visión estratégica que comparta los desafíos y las oportunidades que presenta el territorio.

No obstante, debido a la poca preparación o entrenamiento en el manejo adecuado de conflictos por parte los actores locales, no es sorprendente que las experiencias respecto a las consecuencias en su tratamiento hayan sido las menos deseadas (resultados pobres, desgaste en las relaciones, gran inversión emocional, etc.), lo que ha generado una visión negativa de los conflictos, como algo desagradable y no deseado. Por este motivo es que muchas veces preferimos evitarlos y esperar a que sea realmente necesaria nuestra intervención (conducta pasiva frente al conflicto) y no respondemos proactivamente. Es decir, no buscamos generar espacios de diálogo donde podamos resolver las diferencias existentes, prever las futuras y crear mecanismos que generen un acercamiento de los actores frente a nuevas situaciones de conflicto (Guzmán, 2016).

$\mathrm{Al}$ otro extremo de este continuum de participación social, el diálogo multiactor para la acción colectiva, bajo un enfoque territorial, responde a un proceso de (auto)gobernanza incluyente que apuesta por fortalecer la confianza, normas de reciprocidad, redes para la participación civil, y generación de reglas formales e informales, ya que entiende que sin la construcción de capital social en territorios que albergan proyectos de inversión a gran escala, la reducción o transformación de conflictos socioambientales para el desarrollo sustentable del territorio no es posible. Este diálogo se basa en reglas de funcionamiento para la implementación de procesos ordenados de transferencia de información (disminución de la asimetría), asesoría técnica, mecanismos 
alternativos de resolución de conflictos, entre otros, propiciados por los mismos actores territoriales. Este ejercicio se sustenta en la adquisición de compromisos que buscan responder a incentivos/expectativas comunitarias (y consecuentemente individuales) para impulsar un desarrollo sustentable del territorio. En el caso de incumplimiento de los compromisos por parte de los actores, estos van perdiendo la confianza de los otros actores territoriales participantes del proceso, los que a su vez pierden acceso a los incentivos generados.

De esta manera, el diálogo multiactor para la acción colectiva busca impulsar cambios culturales para pasar de una estrategia transaccionala una estrategia transformacional, a fin de aumentar el nivel de participación y consolidación de las comunidades, la empresa y el Estado en la (auto)gobernanza para la gestión de conflictos de proyectos industriales de gran impacto a través de la construcción de capital social. Esta deriva en un aumento de la colaboración, el liderazgo, el empoderamiento y la generación de alianzas para la consecución de intereses/expectativas en torno a una planificación de desarrollo sustentable del territorio.

De esta manera, para lograr el desarrollo sostenible de los territorios a partir de procesos de diálogo volcados hacia la acción colectiva, como busca el enfoque territorial de responsabilidad empresarial, se debe reconocer la importancia de las economías intrínsecamente multisectoriales, destacando la importancia de alcanzar objetivos múltiples y la articulación de procesos que den respuesta a las causas temáticas, coyunturales y estructurales de los conflictos en el territorio, a partir de la coordinación entre las políticas macro, sectorial y territoriales. De esta manera, el término "diálogo" debe ser asociado a una intencionalidad particular u objetivos claros, como el de contribuir al desarrollo territorial y a la construcción de ciudadanía. De lo contrario pierde claridad y, consecuentemente, lo expone a las críticas que ven al diálogo como un simple espacio de resolución de conflictos que moviliza estrategias transaccionales a corto plazo para responder a los intereses de los actores con mayor poder e influencia en territorio.

Los aspectos operativos-estratégicos característicos del enfoque territorial de responsabilidad empresarial, mencionados anteriormente, van de la mano con: 1) la necesidad de creación de organizaciones e identificación e implicación activa de personas del territorio, líderes que fortalecerán el proceso de diálogo multiactor, tanto cuando este fluye como cuando se enfrentan desafíos y es necesario reforzar la confianza en el proceso; 2) la necesidad de acudir a un tercer actor facilitador y catalizador que pueda acompañar a los actores locales (al menos en sus primeras etapas) en la consolidación de un marco sólido de diálogo, y mantener activa la intencionalidad de un diálogo estratégico y orientado a la acción colectiva. En este contexto es importante tener objetivos y reglas claras que ayuden a gestionar los problemas de acción colectiva y que fortalezcan el liderazgo. Por esta razón es necesario tener autonomía local para invertir en el desarrollo de capital social requerido para construir el proceso, mantener la estructura de incentivos y monitorear sus resultados. De lo contrario, habrá conflictos por interpretaciones dispares de las reglas y falta a los principios básicos de la (auto)gobernanza, por lo que también es 
importante entender el espacio de diálogo multiactor y acción colectiva como un "órgano" periódico de resolución de conflictos y planificación del desarrollo territorial que lleva tiempo ser reconocido y legitimado por parte de los actores locales.

Finalmente, para que las categorizaciones expuestas en este artículo no contribuyan a caer en el riesgo de encerrar el diálogo en una camisa de fuerza que enterraría su potencial de renovación y transformación, se retoman las palabras de Beuret (2012) quien señala que el diálogo (entendido como concertación) es sobre todo un ejercicio de creatividad que ayuda a construir una inteligencia colectiva de situaciones dadas y luego la explota para innovar. Cooperación, acción colectiva y procesos son conceptos lo suficientemente amplios que permiten que los promotores de esos espacios puedan dejar que su imaginación los guíe hacia las formas de diálogo, transformación de conflictos, análisis de la realidad y acuerdos que mejor se adapten a sus aspiraciones y las de sus territorios.

\section{REFERENCIAS}

Arratibel, H., Calvo, G., Estrada, L., Guio, S. y Romero, M. (2019). Guía para la implementación de Procesos de Diálogo y Acción Colectiva bajo un enfoque de Responsabilidad Territorial Empresarial. Bogotá: Insuco, Colombia

Beuret, J. E. y Cadoret, A. (2011). Retour d'expériences sur la concerta-tion vue par les élus locaux et les acteurs environnementaux. Paris: ADEME.
Beuret, J. E. (2006). La conduite de la concertation, pour la gestion de l'envi-ronnement et le partage des ressources. Paris: L'Harmattan.

Beuret, J. E. (2012). Mieux définir la concertation: du pourquoi au comment. Négociations, 1(17), 5.

Bourque D. (2008). Concertation et partenariat. Entre levier et piège du développement des communautés. Montréal: Presses de l'Université du Québec.

Bowen, F., Newenham-Kahindi, A. y Herremans, I. (2010). When suits meet roots: The antecedents and consequences of community engagement strategy. Journal of Business Ethics, 95(2), 21.

Calvo, G. y Carvallo, P. (2015). De la Responsabilidad Social a la Responsabilidad Territorial Empresarial. Tercera conferencia internacional en responsabilidad social en mineria, 1(1), 13.

Cebrián, A. (2004). Génesis, método y territorio del desarrollo rural con enfoque local. Papeles de Geografia, (38), 15.

Chambat, P. y Fourniau, J. M. (2001). Débat public et participation démocratique. En S. Vallemont (dir.). Le débat public: une réforme dans l'État. Paris: LGDJ.

Damonte, G., Glave, M. y Kuramoto, J. (2014). Industrias extractivas y manejo de conflictos. Lima: Grupo de Análisis para el Desarrollo.

De la Cuadra, F. (2015). Indigenous people, socio-environment conflict and post-development in Latin America. Ambiente \& Sociedade, XVIII(2), 23-40.

Dupont, C. (2006). Coopérer pour s'entendre ou s'affronter pour vaincre? Négociations, 5(1), 21.

Dziedzicki, J. M. y Michel, L. (2017). Le tiers-garant de la concertation entre participation publique et gouvernance territoriale. Revue Canadienne de Sciences régionales/Canadian Journal of Regional Sciences, 40(2), 14. 
Fondo para el logro de los ODM (2010). Guía para la transformación de conflictos socioambientales. Lima: ProDiálogo.

GDIAM (2018). Propuesta para una visión compartida sobre la minería en Colombia. Bogotá: Ford Foundation y Banco Interamericano de Desarrollo.

González, A. (coord.) (2013). Los retos de la gobernanza minero energética. Revista Ópera, (14) 7.

González, A. (2016). Profundización, Responsabilidad Social Empresarial y desarrollo local en el sector minero-energético. (Maestría en Responsabilidad Social y Sostenibilidad). Universidad Externado de Colombia, Bogotá.

Guzmán, J. (2016). El conflicto social y la crisis. Naturaleza y dinámica del conflicto social. Asignatura Gestión de riesgos y conflictos, Universidad Continental, 25.

Helin, J. C. (2001). La concertation en matière d'aménagement. Simple obli- gation procédurale ou changement de culture? Annuaire des collectivités locales, (21), 13.

Jorquera, D. (2011). Gobernanza para el desarrollo local. Proyecto Conocimiento y cambio en pobreza rural $y$ desarrollo-Rimisp, (6), 29.

Lederach, J. P. (1996). Mediación. Gernika Gogoratuz, (8), 17.

Lederach, J. P. (2007). Construyendo la paz: Reconciliación sostenible en sociedades divididas (2 ed.). Bilbao: Bakeaz y Gernika Gogoratuz.

Mermet, L. (1998). Place et conduite de la négociation dans les processus de décision complexes: l'exemple des conflits d'environnement. La négociation, situations et problématiques. Paris: Nathan.
Mermet, L. (2006). La “concertation": un terme flottant pour un domaine mouvant? Négociations, 5(1), 4.

Ostrom, E. (2003). Una perspectiva del capital social desde las ciencias sociales: capital social y acción colectiva. Revista Mexicana de Sociologia, 65(1), 78.

Pruitt, D., Rubin, J. y Kim Sung, H. (1994). Social Conflict. Escalation, Stalemate, and Settlement. New York: McGraw-Hill.

Schejtman, A. y Berdegué, J. (2003). Desarrollo territorial rural. Debates y temas rurales-RIMISP, (1), 53.

Sepúlveda, S., Rodríguez, A., Echeverri, R. y Portilla M. (2003). El enfoque territorial del desarrollo rural. San José: Instituto Interamericano de Cooperación para la Agricultura.

Tapia, G., Lumerman, P., Portilla, J. y Spadoni, E. (2009). Manual conflicto y desarrollo. Enfoque sensible al conflicto para organizaciones de desarrollo. Buenos Aires: Fundación Cambio Democrático.

Walton, R. y Mac Kersie, R. (1965). A Behavioral Theory of Labor Negocia-tions. An Analysis of a Social Interaction System. Ithaca: ILR Press.

Watkins, G. (coord.) (2017). Lecciones de cuatro décadas de conflicto en torno a los proyectos de infraestructura en América Latina y el Caribe. Monografías del Banco Interamericano de Desarrollo. Washington: BID. 